\title{
Artificial intelligence cooperation to support the global response to COVID-19
}

\author{
In an unprecedented effort of scientific collaboration, researchers across fields are racing to support the response \\ to COVID-19. Making a global impact with AI tools will require scalable approaches for data, model and code \\ sharing; adapting applications to local contexts; and cooperation across borders.
}

\begin{abstract}
Miguel Luengo-Oroz, Katherine Hoffmann Pham, Joseph Bullock, Robert Kirkpatrick, Alexandra Luccioni, Sasha Rubel, Cedric Wachholz, Moez Chakchouk, Phillippa Biggs, Tim Nguyen, Tina Purnat and Bernardo Mariano
\end{abstract}

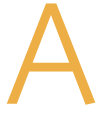
s of 24 April 2020, the SARS-CoV-2 virus had infected 823,626 people with more than 40,598 confirmed deaths, and with nearly every citizen of the planet at risk ${ }^{1}$. Given the need to interrupt disease transmission and the increasing scale of the pandemic, digital technologies are relevant to support the fight against the virus. Among these technologies, machine learning and artificial intelligence (AI) methods are being applied in many COVID-related domains, resulting in approximately 200 preprints and scientific articles from 1 January 2020 to 24 April $2020^{2}$. However, for AI applications addressing COVID-19 to have a global impact, large-scale data and model sharing, operational validation, and adaptation to local contexts are needed. This requires cooperation and solidarity across borders as well as the involvement of many relevant parties, including healthcare workers.

\section{Taxonomy and applications}

In a review of the emerging literature at the intersection of AI and COVID-19 by some of $\mathrm{us}^{3}$, a framework to categorize multidisciplinary research on three scales is proposed: molecular, clinical and societal (epidemiology and infodemics).

Molecular applications include protein structure prediction ${ }^{4}$, improving viral nucleic acid testing ${ }^{5}$, drug repurposing ${ }^{6}$ and drug discovery ${ }^{7}$. These applications draw on a range of AI methods including the construction and mining of biomedical knowledge graphs, deep learning architectures designed to predict characteristics of protein structures or protein-ligand binding affinities, text modelling approaches applied to genetic sequences, and the use of reinforcement learning for drug design.

Clinical applications to improve patient care range from diagnosis to patient follow-up, treatment and outcome prediction. Deep learning techniques can help image-based diagnosis of COVID-19 from patterns in X-ray images and computed tomography (CT) scans ${ }^{8}$. Operational human-in-the-loop AI systems have been developed to help radiologists reduce the time needed to review scans. Smart watches, mobile phones and other wearables can potentially be used for disease tracking 9 , along with other remote monitoring applications that allow doctors to follow patients remotely, saving time and personal protective equipment. Combining different data sources like health records and medical imagery can help predict patient outcomes ${ }^{10}$ and could be used to project the needs of hospitals and healthcare systems such as surges in demand for intensive care unit beds. The use of robotics in telemedicine and in other tasks like cleaning and sterilizing medical facilities could also prove valuable.

For epidemiology, AI can improve early warning systems and the modelling of empirical data ${ }^{11}$. In particular, AI can be applied to understand and simulate public policy interventions such as quarantining, social distancing and de-escalation strategies, and augment classical epidemiological techniques by approximating functions for which analytic transmission equations are not known ${ }^{12,13}$. Additionally, unsupervised clustering techniques and risk-scoring algorithms, which are able to combine disparate data sources, may help identify similarities between regions, and forecast which areas will require more resources.

For infodemics, AI can be used to fight misinformation and disinformation by managing the current overabundance of information, which creates confusion and makes it difficult to identify trusted sources $^{14,15}$. AI methods can support audience analysis (for example, social media,
$\mathrm{TV}$, radio) and accelerate fact checking ${ }^{16}$. Social media analysis, for example, is providing insights into global trends and sentiment around the pandemic and its socioeconomic impacts ${ }^{17}$. Research is being conducted to identify the emergence and escalation of hate speech and verbal attacks against minorities and communities suffering from discrimination that may result in violent action or their exclusion from access to healthcare ${ }^{18}$. Virtual assistants and chatbots are being used to disseminate trusted content at scale ${ }^{19}$, requiring mechanisms for propagation of updates to information as new evidence becomes available in the COVID-19 response.

\section{Data and model sharing}

AI applications require data. There are currently dozens of data-sharing initiatives around COVID-19 at the three application scales, spanning the international, national and local levels. These datasets include: genetic sequences ${ }^{20}$, genome analysis ${ }^{21}$, protein structures, patient clinical data, medical imagery, case statistics, epidemiological data ${ }^{22}$, mobility data, social media posts, news articles and scientific literature ${ }^{23}$. The hyper-fragmentation of data-sharing efforts is a challenge, as it might result in advances that are restricted to particular projects and local communities. Establishing scalable approaches for data, model and code sharing could accelerate the development and dissemination of new applications. At this stage, global, open, comprehensive, comparable and verified data-sharing initiatives will be beneficial to connect and promote cooperation between disparate communities and geographies ${ }^{24}$.

Open science can accelerate knowledge dissemination and capacity building of national health systems, supported by multi-stakeholder AI partnerships operating across international borders. For example, the data platform of the 
Epidemic Intelligence from Open Sources $(\text { EIOS })^{25}$ network enables early detection, verification and assessment of public health risks and threats using open source information. Under the philosophy of collaboration and not competition in early detection, the community of practice for public health intelligence includes governments, international organizations and research institutes that collaboratively assess and share information about outbreak events in real time. The EIOS network picked up the first article reporting an unknown pneumonia cluster in Wuhan at 3:14 UTC on 31 December 2019. From the epidemiological perspective, global standards and interoperability between databases could enable coordinated response and decision-making at global, national and local levels. Understanding the epidemiologic features and risk characteristics of different demographics as we move into different phases of the pandemic will require accounting for health system resource capacities, public health interventions, environmental factors and the social implications of COVID-19.

Beyond data sharing, few initiatives currently share trained AI models related to any of the proposed applications. Barriers to be overcome include constraints due to specific computational, architecture and infrastructure requirements; a lack of documentation; validation and explainability aspects; and legal concerns regarding privacy and intellectual property. Sharing pre-trained and validated AI models could accelerate the adaptation of solutions to local contexts. Examples of models that could be widely applicable include those used to diagnose illness from imagery, predict patient outcomes, filter misinformation and disinformation based on spreading patterns through social networks, or distil knowledge graphs from large collections of scientific articles. Getting to a future of open AI models will require AI governance frameworks that take into account ethical, social, clinical, legal and commercial considerations.

\section{From research to impact}

One of the challenges of making a greater impact with AI applications is not knowing where to start and how efforts can be most effective. There is a need for greater cooperation between practitioners and the AI community. The AI community already is, and should continue to seek advice and guidance from domain experts, including government officials, healthcare professionals and first responders, among others. They can help flag processes that are good candidates for automation.
These include repetitive or time-consuming tasks that involve finding patterns in rich image, video, audio or biomedical data - for example, CT scans - or tasks that involve synthesizing massive datasets from sources such as symptom tracking systems or social media channels.

To date, few of the research projects and systems presented are as yet sufficiently mature to be operationalized at scale, with different applications having different timescales of development, validation and deployment. As a result, it is important for the founders, users and AI community to map which technologies could assist with the short-term response, mid-term recovery efforts, and the longer-term preparedness for future pandemics.

Developing solutions for the current pandemic requires coordination and prioritization $^{26}$, focused on key unmet needs that account for operational realities. When possible, solutions should be based on adapting already validated systems ${ }^{27}$ and should avoid overburdening an already stretched healthcare workforce with new tools that may not always help to improve outcomes. Furthermore, it is important that new and existing solutions are adapted to fill the specific needs and contexts of low- and middle-income countries. Applications dealing with clinical and patient data should not ignore the existing regulatory frameworks designed to ensure quality, security and safety, and minimize potential risks and harms. Despite the urgency of the fight against COVID-19, rigorous assessment of potential solutions is required, which in some opportunities can be fast-tracked without increasing risks.

Collaborations among global health actors, private sector partners and other stakeholders are already forming to accelerate the development, production and equitable global access to new health technologies essential to COVID-1928. Coordination and cooperation between regulatory bodies and international and regional organizations would aid in channelling research on the ground, and would assist in implementing systems in countries with weaker frameworks and governance structures. Compliance with reporting guidelines ${ }^{29,30}$ would enable more systematic and efficient reviews of evidence, and comparisons of healthcare-related AI applications along the dimensions of safety, clinical validity and performance accuracy.

The global fight against the COVID-19 infodemic should be treated as a scientific challenge on a par with the spread of the disease itself, since behavioural change is critical to the pandemic response. Media and information literacy remain critical, and there is a need to quantify individual rates of exposure to misinformation and disinformation. Infodemic management frameworks can be used to support timely translation of evidence into knowledge and advocacy adapted to local cultures, languages and contexts. AI methods and tools should be used to fill the health information gaps for individuals and policymakers alike for a whole-of-society response based on evidence and science. To effectively combat the infodemic while ensuring the fundamental right to access to information, the international community must share and amplify good practices, identify priorities, facilitate partnerships, and provide advice and technical assistance to governments and relevant national stakeholders to foster international cooperation. The pandemic is stoking xenophobia, hate and exclusion, posing a far-reaching - and potentially long-lasting - threat to human rights ${ }^{31}$. Understanding the dynamics and spread of hateful speech will help design more effective interventions and change the narrative.

\section{Global challenges and digital cooperation}

We now have the ability to create new AI tools to address age-old problems. But having the means to create such applications doesn't necessarily mean we should. Any AI application that could be used in the fight against COVID-19 ought to undergo an assessment to ensure that it complies with ethical principles and, above all, respects human rights. Even when following privacy-by-design and ethics-by-design guidelines in the development and deployment of AI-based solutions, practitioners will be confronted with questions on how to protect fundamental rights, including the right to privacy. When rolling out any of the above proposed AI solutions at scale, we must ensure they do not breach global commitments to protect rights and freedoms, including commitments to non-discrimination, preventing mass surveillance, and protecting journalistic sources. In order to do so, stakeholders should ensure that principles, including those of openness and accessibility, are at the heart of AI-enabled solutions. AI applications in health should also promote fair and equitable global access, supporting member states' commitments to achieve universal health coverage.

The global nature of the pandemic requires global solutions, but there is also a need for local adaptation to account for biases and contextual factors. For instance, 
a system to diagnose COVID-19 pneumonia in chest X-rays will need to be trained differently in countries with different levels of prevalence of diseases or conditions with related image patterns, such as tuberculosis and HIV. Similarly, epidemiological models tailored to pastoral areas, island states, refugee camps or conflict zones should take into account different socioeconomic, cultural and structural variables than the ones described in scientific literature which have been developed mostly in China or in Western countries. Likewise, chatbots to inform citizens about public health measures require specific natural language processing models, and often vast amounts of training data, which today are available for just a few hundred of the more than 7,000 living languages. The participation of researchers and local data science collaboratives from underrepresented regions including Africa and Latin America, for example the Data Science Africa network, will be critical to ensure that any AI system has a positive impact at global scale. With this in mind, solutions may need to be designed with low-bandwidth requirements up front - for example, medical diagnosis apps without need for connectivity and that do not compromise privacy or data security. Such applications represent an opportunity to promote edge computing for AI and other energy-efficient approaches for the responsible use of computational resources.

The role of AI in providing meaningful solutions to the current pandemic has yet to be defined. However, there is a clear need for a globally inclusive research agenda to direct action against this pandemic - and future ones - without leaving anyone behind. It is reasonable to believe that the world will be more digital than ever in the post-coronavirus era, and that AI will increasingly become one of the driving forces shaping our society ${ }^{32}$. This pandemic underscores the urgency for stakeholders to effectively translate ethical principles for $\mathrm{AI}$ into practice ${ }^{33}$. 'Solidarity' is the name of the international clinical trial launched by the World Health Organization to help find an effective treatment for COVID-19 ${ }^{34}$. It is the sense of solidarity with our neighbours that encourages physical distancing, which is vital to suppressing transmission of the virus within communities. Similarly, solidarity should be a core principle guiding the development of innovative and ethical AI applications to fight the COVID-19 pandemic, and help advance the global Sustainable Development Agenda ${ }^{35}$.

\section{Miguel Luengo-Oroz (D) 1 , \\ Katherine Hoffmann Pham' \\ Joseph Bullock (D)', Robert Kirkpatrick', \\ Alexandra Luccioni (D)2 ${ }^{2}$, Sasha Rubel ${ }^{3}$, \\ Cedric Wachholz $z^{3}$, Moez Chakchouk ${ }^{3}$, \\ Phillippa Biggs ${ }^{4}$, Tim Nguyen (D) \\ Tina Purnat (D) ${ }^{5}$ and Bernardo Mariano ${ }^{5}$}

${ }^{1}$ United Nations Global Pulse, New York, NY, USA.

${ }^{2}$ Mila, Université de Montréal, Montreal, Quebec,

Canada. ${ }^{3}$ United Nations Educational, Scientific and

Cultural Organization, Paris, France. ${ }^{4}$ International

Telecommunications Union, Geneva, Switzerland.

${ }^{5}$ World Health Organization, Geneva, Switzerland.

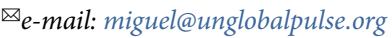

Published online: 22 May 2020

https://doi.org/10.1038/s42256-020-0184-3

\section{References}

1. Coronavirus disease (COVID-2019) situation reports. WHO https://www.who.int/emergencies/diseases/ novel-coronavirus-2019/situation-reports (2020).

2. UNGlobalPulse/covid19-literature-search. GitHub https://github com/UNGlobalPulse/covid19-literature-search (2020).

3. Bullock, J., Luccioni, A., Hoffmann Pham, K., Lam, C. S. N. \& Luengo-Oroz, M. Preprint at https://arxiv.org/abs/2003.11336 (2020).

4. Senior, A. W. et al. Nature 577, 706-710 (2020).

5. Lopez-Rincon, A. et al. Preprint at https://doi. org/10.1101/2020.03.13.990242 (2020)

6. Richardson, P. et al. Lancet 395, E30-E31 (2020)

7. Zhavoronkov, A. et al. Preprint at https://doi.org/10.26434/ chemrxiv.11829102.v2 (2020).

8. Shi, F. et al. IEEE Rev. Biomed. Eng. https://doi.org/10.1109/ RBME.2020.2987975 (2020).

9. Radin, J., Wineinger, N. E., Topol, E. J. \& Steinhubl, S. R. Lancet Dig. Health 2, E85-E93 (2020).

10. Shi, W. \& Peng, X. Preprint at https://doi.org/10.2139/ ssrn.3546089 (2020).

11. Al-qaness, M. A. A., Ewees, A. A., Fan, H. \& Abd El Aziz, M. J. Clin. Med. 9, 674 (2020).
12. Nuria, O. et al. Sci. Adv https://doi.org/10.1126/sciadv.abc0764 (2020).

13. Dandekar, R. \& Barbastathis, G. Preprint at https://arxiv.org/ abs/2003.09403 (2020).

14. Infodemic management: a key component of the COVID-19 global response. WHO https://apps.who.int/iris/ handle/10665/331775 (2020).

15. Coronavirus Disease 2019 (COVID-19) - Situation Report 86 (WHO, 2020).

16. Coronavirus disease 2019 (COVID-19) - Situation Report 100 (WHO, 2020).

17. Gallotti, R. et al. Preprint at https://doi.org/10.1101/2020.04.08 20057968 (2020).

18. Velásquez, N. et al. Preprint at https://arxiv.org/abs/2004.00673 (2020).

19. Sundareswaran, V. \& Firth-Butterfield, K. Chatbots provide millions with COVID-19 information every day, but they can be improved - here's how. World Economic Forum https://www. weforum.org/agenda/2020/04/chatbots-covid-19-governance-imp roved-here-s-how/ (2020).

20. Cohen, J. Science https://www.sciencemag.org/news/2020/01/ chinese-researchers-reveal-draft-genome-virus-implicatedwuhan-pneumonia-outbreak (2020)

21. Hadfield, J. et al. Bioinformatics 34, 4121-4123 (2018).

22. Zastrow, M. Nature https://doi.org/10.1038/d41586-020-00740-y (2020).

23. Wang, L. L. et al. Preprint at https://arxiv.org/abs/2004.10706 (2020).

24. Teran, J. UN Department of Economic and Social Affairs https:// covid-19-response.unstatshub.org/open-data/hdx-making-covid 19-data-accessible/ (2020).

25. Epidemic intelligence from open sources. WHO https://www. who.int/eios (2020).

26. Digital health. WHO https://www.who.int/health-topics/ digital-health (2020).

27. Topol, E. J. Nat. Med. 25, 44-56 (2019).

28. Access to COVID-19 Tools (ACT) Accelerator (WHO, 2020).

29. The CONSORT-AI and SPIRIT-AI Steering Group Nat. Med. $\mathbf{2 5}$, 1467-1468 (2019).

30. Collins, G. S. \& Moons, K. G. M. Lancet 393, 1577-1579 (2019).

31. Press briefing note on Americas/prison conditions. UN Human Rights https://shar.es/aHIzht (2020).

32. The Age of Digital Interdependence (UN Secretary General's High-level Panel on Digital Cooperation, 2019).

33. Jobin, A., Ienca, M. \& Vayena, E. Nat. Mach. Intell. 1 , 389-399 (2019).

34. "Solidarity" clinical trial for COVID-19 treatments. WHO https:// www.who.int/emergencies/diseases/novel-coronavirus-2019/ global-research-on-novel-coronavirus-2019-ncov/ solidarity-clinical-trial-for-covid-19-treatments (2020). 35. Vinuesa, R. Nat. Commun. 11, 233 (2020).

Acknowledgements

The authors who are staff members or employees of international organizations (M.L.-O., K.H.P., J.B., R.K., S.R., C.W., M.C., P.B., T.N., T.P. and B.M.) are responsible for the views expressed in this paper and they do not present the views of their organizations. The authors would like to acknowledge S. Kuku, F. Vacarelu and C. Sin Nga Lam for their advice and support in the preparation of this manuscript.

\section{Competing interests}

The authors declare no competing interests. 